\title{
Endophthalmitis following extracapsular cataract surgery: a review of 32 cases
}

\author{
C J Heaven, P J Mann, D L Boase
}

\begin{abstract}
Thirty two cases of endophthalmitis following extracapsular cataract surgery that had occurred within our department and had undergone intraocular diagnostic tap between May 1982 and May 1991 were reviewed. An infectious agent was identified in 20 cases $(62 \cdot 5 \%)$. The commonest organism was Staphylococcus epidermidis (11 cases) (55\%). Proteus was the only gram negative organism identified (four cases) (20\%). Both of these organisms were associated with a favourable visual outcome. In the culture positive subgroup 15 eyes $(75 \%)$ achieved a final acuity of $6 / 60$ or better with 10 eyes $(50 \%)$ gaining $6 / 12$ or better. Thirteen $(65 \%)$ of the culture positive cases were managed without vitreal intervention. Of these $11(85 \%)$ achieved $6 / 60$ or better with eight $(62 \%)$ gaining $6 / 12$ or better. It appears that when an endophthalmitis follows uncomplicated extracapsular cataract surgery delivery of antibiotic by the 'conventional' routes (topical, subconjunctival and systemic) is consistent with a favourable visual result in many cases. A modified anterior chamber diagnostic tap technique is described.
\end{abstract}

Bacterial endophthalmitis is a potentially catastrophic complication of cataract surgery. Its management remains controversial. Dissatisfaction with the visual outcome from 'conventional' treatment using topical, subconjunctival, and systemic antibiotic has resulted in a trend towards the use of intraocular antibiotic injection and vitrectomy. ${ }^{1-8}$ However this evolution in management has been largely based on the published experience of endophthalmitis following intracapsular cataract surgery and endophthalmitis occurring after other forms of ocular surgery or trauma. ${ }^{2359-14}$ There are relatively few reports of the visual outcome of endophthalmitis specifically following extracapsular cataract surgery. ${ }^{15}$ We have therefore undertaken a review of cases of endophthalmitis occurring within our department following planned extracapsular cataract extraction.

\section{Patients and methods}

Operating theatre records were used to trace cases that had suffered an endophthalmitis following extracapsular cataract surgery and who had undergone anterior chamber and/or vitreous diagnostic tap. Thirty two such cases had occurred between May 1982 and May 1991. The medical records of each case were reviewed and the information extracted included: patient personal details, details of the cataract surgery, time of onset of endophthalmitis and presence of hypopyon, details of the intraocular diagnostic tap, additional surgical management, use and route of administration of antibiotics and corticosteroids, duration of intraocular inflammation, preoperative and final visual acuity, period of follow-up, and other co-existent ocular pathology.

In all cases the intraocular diagnostic tap specimen had been cultured on blood agar, chocolate agar, Sabouraud's media, and thioglycollate broth. A microbiological report was available for each case.

\section{Results}

In 20 cases (62.5\%) a bacterial infection had been identified (culture positive). The spectrum and relative frequency of organisms is shown in Figure 1 and was similar to that reported in previous series. ${ }^{2357-1316} \mathrm{~A}$ gram positive organism was identified in 16 cases $(80 \%)$. All four cases (20\%) of gram negative infection were due to Proteus species. The commonest organism was Staphylococcus epidermis, in 11 cases (55\%). Twelve cases $(37 \cdot 5 \%)$ were culture negative. The culture positive and culture negative cases are subsequently considered as separate groups.

GROUP 1: CULTURE POSITIVE CASES

Individual case details are shown in Table 1. Of the 20 culture positive cases of endophthalmitis 12 were female and eight male. The age range was 41 to 91 years with a mean of 77 years. In nine the cataract surgery had been performed under general anaesthesia, while 11 had received a local anaesthetic of which five had been day cases. Nineteen of the 20 (95\%) had undergone 'uncomplicated' extracapsular cataract extraction with posterior chamber intraocular lens implantation (ECCE $+\mathrm{P} / \mathrm{C} \mathrm{IOL})$. In a single case (case 1) a planned ECCE $+\mathrm{P} / \mathrm{C}$ IOL had been complicated by vitreous loss. This had necessitated an anterior vitrectomy and conversion to the use of an anterior chamber intraocular lens.

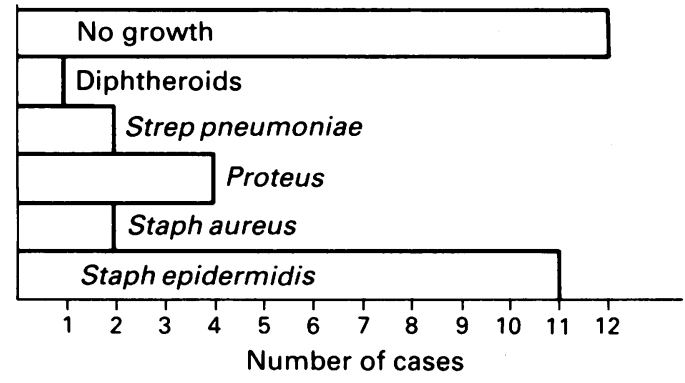

Figure 1 Incidence and spectrum of organisms identified from intraocular tap - all cases. 
Table 1 Endophthalmitis after extracapsular cataract surgery. Culture positive cases

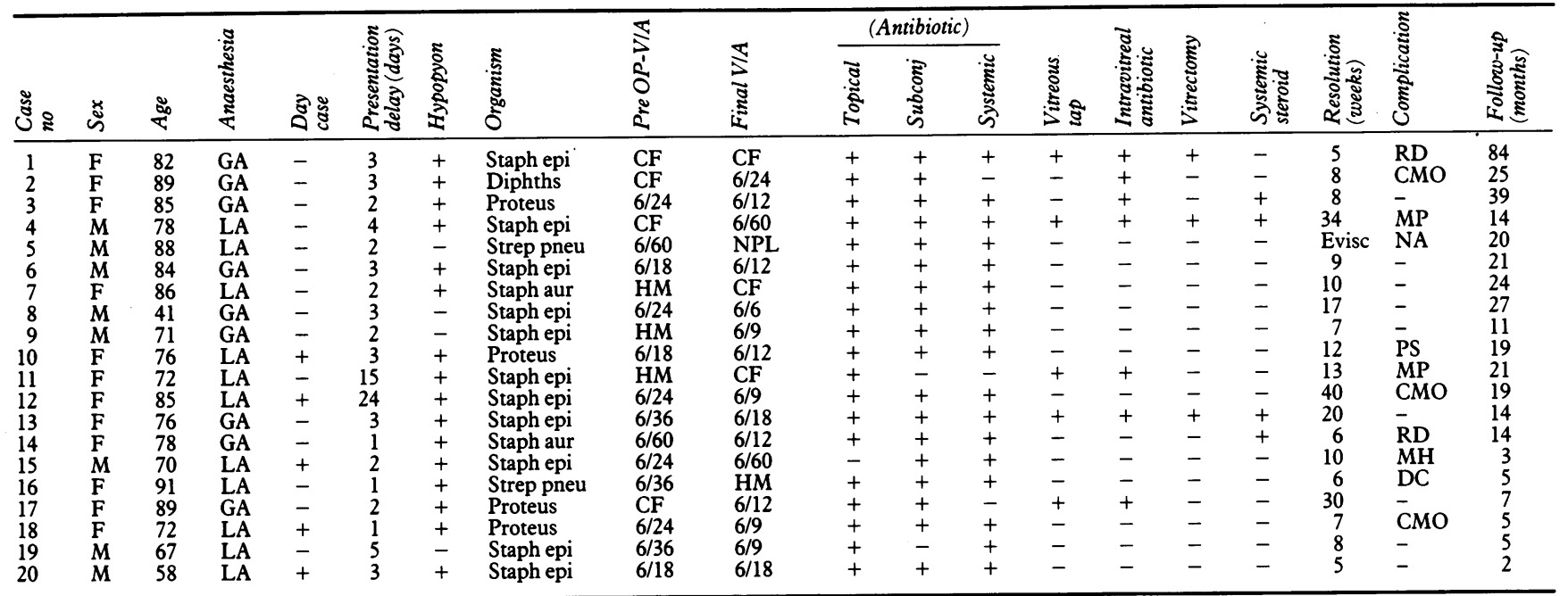

$\mathrm{GA}=$ general anaesthetic; $\mathrm{LA}=$ local anaesthetic; Staph epi=Staphylococcus epidermidis; Staph aur=Staphylococcus aureus; Strep pneu=Streptococcus pneumoniae; Diphths=Diphtheroids; $\mathrm{RD}=$ retinal detachment; $\mathrm{CMO}=$ cystoid macular oedema; $M \mathrm{P}=$ macular pucker; $\mathrm{PS}=$ pupillary seclusion; $M H=$ macular hole;

$\mathrm{DC}=$ decompensated cornea; $\mathrm{NA}=$ not applicable.

The time interval between cataract surgery and diagnosis of endophthalmitis (presentation delay) ranged from 1 to 24 days with a mean of 4 days. Following the onset of clinical endophthalmitis all cases underwent a diagnostic anterior chamber tap. In addition five had a pars plana vitreous tap. Three cases underwent pars plana vitrectomy. Seven cases received intravitreal antibiotic. In four of these gentamicin was used alone while three received a combination of antibiotics; (case 2: gentamicin+penicillin+ methicillin, case 4: gentamicin+methicillin, case 13: gentamicin + cefuroxime + vancomycin). The dose of intravitreal gentamicin ranged from $0.1 \mathrm{mg}$ to $0.3 \mathrm{mg}$. In no case was the intraocular lens removed. The duration of intraocular inflammation ranged from 5 to 40 weeks, mean 13 weeks. Follow-up was for between 2 and 84 months, mean 19 months.

Figure 2 is a scattergram which compares Figure 2 Scattergramculture positive cases, preoperative versus final visual acuity.

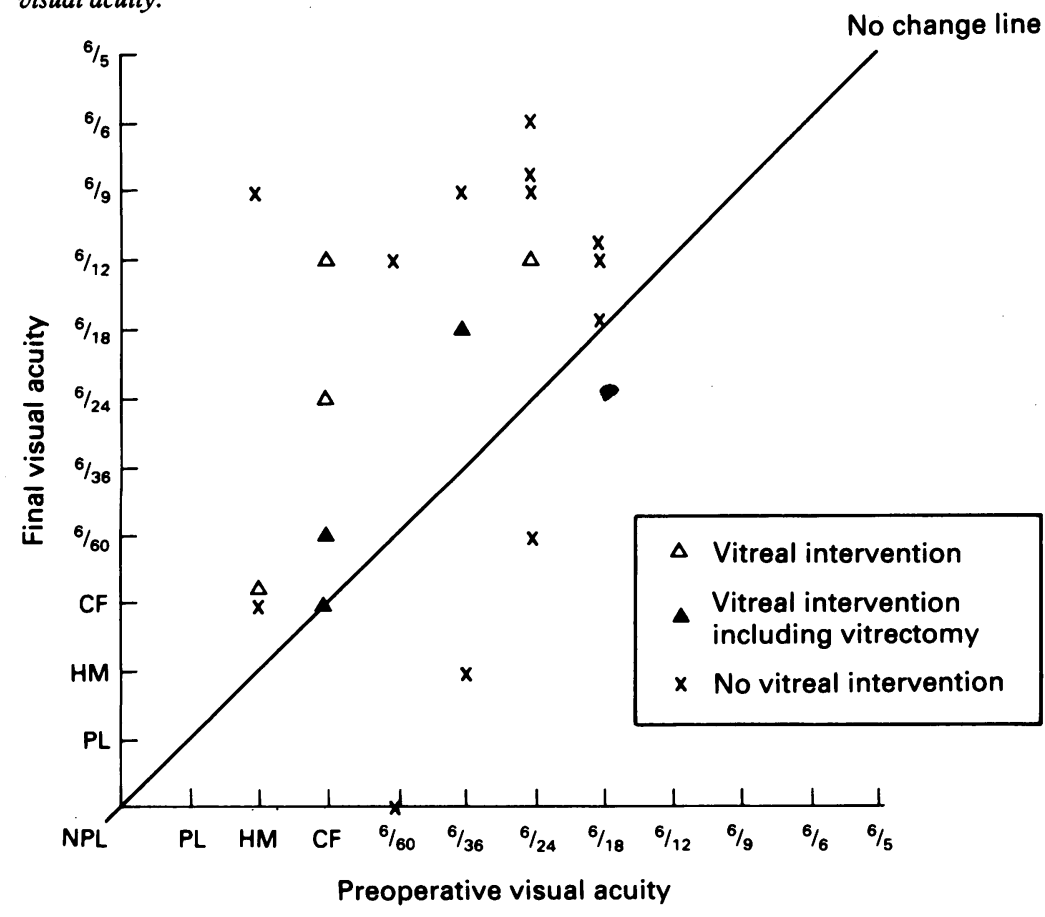

culture positive group. In 17 cases (85\%) the final vision was as good as or better than the preoperative acuity. Fifteen $(75 \%)$ had a final acuity of $6 / 60$ or better. Ten $(50 \%)$ had a final acuity of $6 / 12$ or better. A favourable outcome was associated with Proteus and Staphylococcus epidermidis infection and no vitreal intervention. Only one of the five vitreous taps and two of the seven instances of intravitreal antibiotic injection gained an acuity of $6 / 12$ or better.

Five cases had a 'poor' visual outcome with final acuity less than $6 / 60$. This tended to be associated with Streptococcus pneumoniae infection. ${ }^{10}$ The two cases of Staphylococcus epidermidis endophthalmitis whose final acuity was worse than $6 / 60$ had both undergone vitreous tap and intravitreal antibiotic and both had suffered ocular morbidity that could have been related to this intervention, namely retinal detachment and macular pucker.

\section{GROUP 2: CULTURE NEGATIVE CASES}

Individual case details are shown in Table 2. Of the 12 culture negative cases seven were female and five male. The age range was 47 to 87 years, mean 68 years. In nine cases the cataract surgery was performed under general anaesthesia, while three received a local anaesthetic of which two had been day cases. In all 12 cases the extracapsular cataract surgery was 'uncomplicated' with the insertion of a posterior chamber intraocular lens. Ten cases suffered an endophthalmitis within 8 days of cataract surgery, mean 5 days. In all of these cases the intraocular inflammation eventually subsided taking from 6 to 32 weeks to do so, mean 12 weeks. However, in two cases (cases 27 and 30) the endophthalmitis was of late onset (90 and 44 days respectively) and in both the intraocular inflammation persists after prolonged follow-up. These two cases appear to have behaved in a manner typical of the recently described 'localised endophthalmitis' where microorganisms may be sequestered within the capsular bag resulting in negative diagnostic tap. ${ }^{617}$ 
Table 2 Endophthalmitis after extracapsular cataract surgery. Culture negative cases

\begin{tabular}{|c|c|c|c|c|c|c|c|c|c|c|c|c|c|c|c|c|c|c|}
\hline \multirow[b]{2}{*}{ రั้ } & \multirow[b]{2}{*}{ ふ઼ } & \multirow[b]{2}{*}{$\underset{+}{\$}$} & \multirow{2}{*}{ 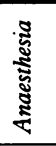 } & \multirow[b]{2}{*}{ 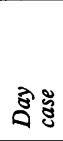 } & \multirow{2}{*}{ 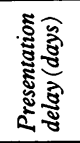 } & \multirow[b]{2}{*}{ స్ } & \multirow{2}{*}{$\begin{array}{l}5 \\
5 \\
0 \\
0 \\
2 \\
2\end{array}$} & \multirow[b]{2}{*}{ 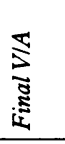 } & \multicolumn{3}{|c|}{ (Antibiotic) } & \multirow[b]{2}{*}{ 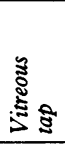 } & \multirow{2}{*}{ 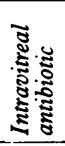 } & \multirow[b]{2}{*}{ 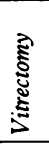 } & \multirow[b]{2}{*}{ 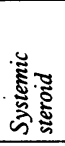 } & \multirow[b]{2}{*}{ 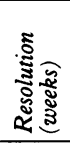 } & \multirow{2}{*}{ 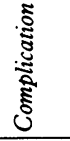 } & \multirow[b]{2}{*}{ 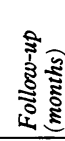 } \\
\hline & & & & & & & & & 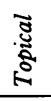 & 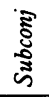 & 胥 & & & & & & & \\
\hline $\begin{array}{l}21 \\
22 \\
23 \\
24 \\
25 \\
26 \\
27 \\
28 \\
29 \\
30 \\
31 \\
32\end{array}$ & $\begin{array}{l}F \\
F \\
F \\
F \\
F \\
F \\
F \\
M \\
M \\
M \\
M \\
M\end{array}$ & $\begin{array}{l}75 \\
70 \\
87 \\
53 \\
82 \\
57 \\
78 \\
75 \\
63 \\
47 \\
73 \\
53\end{array}$ & $\begin{array}{l}\text { LA } \\
\text { GA } \\
\text { GA } \\
\text { GA } \\
\text { GA } \\
\text { GA } \\
\text { GA } \\
\text { LA } \\
\text { GA } \\
\text { LA } \\
\text { GA } \\
\text { GA }\end{array}$ & $\begin{array}{l}+ \\
- \\
- \\
- \\
- \\
- \\
- \\
+ \\
- \\
- \\
- \\
-\end{array}$ & $\begin{array}{r}4 \\
7 \\
6 \\
6 \\
6 \\
5 \\
90 \\
2 \\
8 \\
44 \\
7 \\
3\end{array}$ & $\begin{array}{l}+ \\
+ \\
+ \\
+ \\
+ \\
+ \\
+ \\
+ \\
+ \\
+ \\
+ \\
+\end{array}$ & $\begin{array}{l}6 / 18 \\
6 / 12 \\
6 / 60 \\
6 / 36 \\
6 / 12 \\
6 / 60 \\
6 / 60 \\
\text { CF } \\
6 / 60 \\
H M \\
6 / 60 \\
6 / 18\end{array}$ & $\begin{array}{l}6 / 9 \\
6 / 9 \\
6 / 9 \\
6 / 9 \\
6 / 9 \\
6 / 6 \\
6 / 18 \\
\text { PL } \\
6 / 5 \\
6 / 9 \\
6 / 18 \\
6 / 60\end{array}$ & $\begin{array}{l}+ \\
- \\
+ \\
+ \\
+ \\
+ \\
+ \\
+ \\
+ \\
+ \\
+ \\
+\end{array}$ & $\begin{array}{l}+ \\
+ \\
+ \\
+ \\
+ \\
+ \\
+ \\
+ \\
+ \\
+ \\
+ \\
+\end{array}$ & $\begin{array}{l}- \\
\overline{+} \\
+ \\
+ \\
+ \\
+ \\
+ \\
+ \\
+ \\
+ \\
+ \\
+\end{array}$ & $\begin{array}{l}- \\
- \\
- \\
- \\
- \\
+ \\
- \\
- \\
- \\
- \\
+\end{array}$ & $\begin{array}{l}- \\
- \\
- \\
+ \\
- \\
- \\
- \\
- \\
- \\
- \\
+\end{array}$ & $\begin{array}{l}- \\
\overline{-} \\
- \\
- \\
\overline{-} \\
+ \\
- \\
- \\
- \\
- \\
-\end{array}$ & $\begin{array}{l}+ \\
+ \\
- \\
- \\
+ \\
+ \\
+ \\
- \\
- \\
- \\
- \\
-\end{array}$ & $\begin{array}{r}6 \\
13 \\
13 \\
13 \\
32 \\
6 \\
\star \\
8 \\
4 \\
\star \\
6 \\
22\end{array}$ & $\begin{array}{l}- \\
- \\
- \\
- \\
- \\
- \\
- \\
- \\
- \\
\overline{M P P} \\
M P\end{array}$ & $\begin{array}{r}10 \\
45 \\
20 \\
55 \\
34 \\
30 \\
30 \\
7 \\
31 \\
60 \\
7 \\
35\end{array}$ \\
\hline
\end{tabular}

^=Ongoing intraocular inflammation.

Figure 3 is a scattergram which compares preoperative and final visual acuities for the culture negative group. Eleven eyes ( $92 \%)$ had a final visual acuity of $6 / 60$ or better. Eight $(67 \%)$ attained a final acuity of $6 / 12$ or better.

The culture negative group thus overall had a better visual outcome than the culture positive group. This is in agreement with previously reported experience..$^{-12}$

\section{Discussion}

Historically the visual outcome from endophthalmitis using the 'conventional' treatment regimen of topical, subconjunctival, and systemic antibiotics was poor. Up to the late 1970 s visual results of only hand movements or worse were reported to occur in $67 \%$ to $96 \%$ of cases. ${ }^{1}$ In an attempt to salvage useful vision in a greater number of eyes direct injection of antibiotic into the vitreous and in some cases vitrectomy has been gaining favour in the management of endophthalmitis. A prospective study to determine the benefit of vitrectomy in endophthalmitis is currently in progress. ${ }^{1}$

Previously reported series of cases of

Figure 3 Scattergramculture negative cases, preoperative versus final visual acuity.

endophthalmitis have considered its occurrence

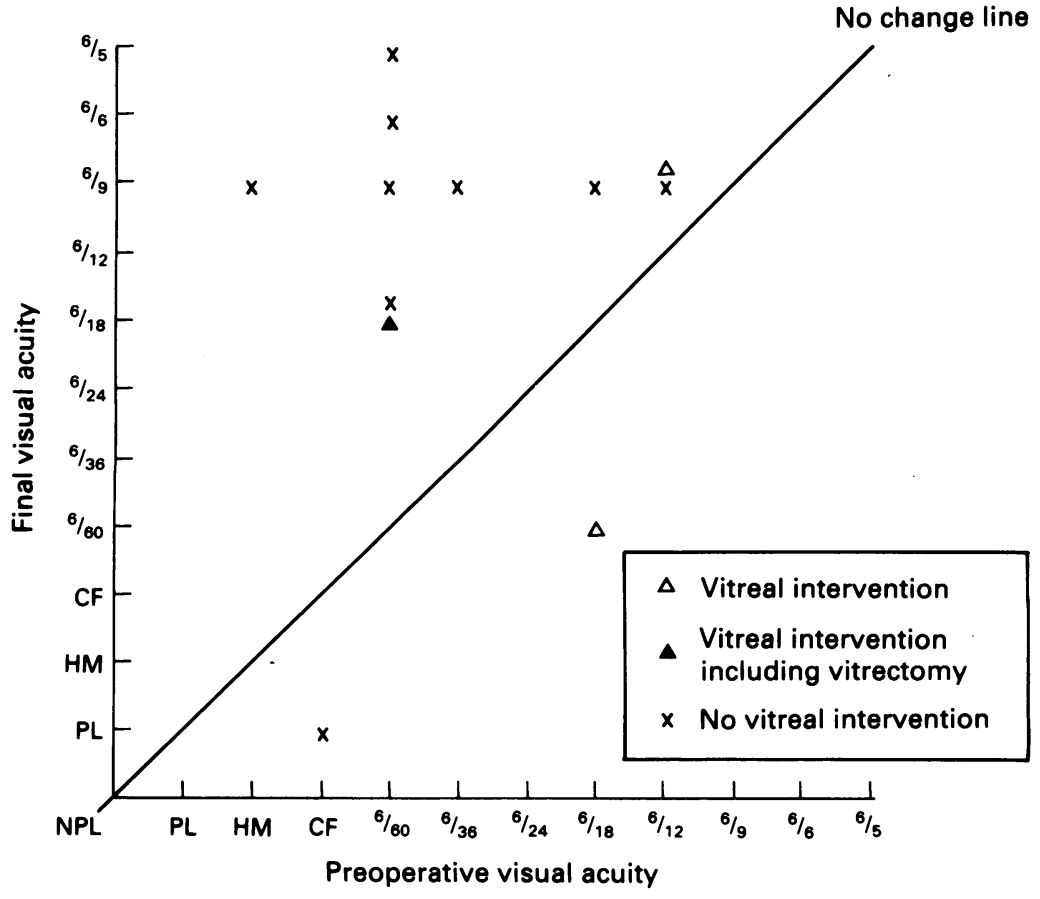

collectively after various forms of ocular surgery and trauma. In this context vitreal intervention has resulted in an improved visual outcome with those eyes gaining an acuity of $20 / 400$ or better reported at between $42 \%$ and $73 \% .^{23579-12.18}$ Fewer eyes achieve a near normal acuity; $13 \%$ $20 / 40$ or better, ${ }^{2} 55 \% 6 / 15$ or better. ${ }^{18}$ However there have been relatively few reports of the visual outcome from endophthalmitis specifically when it occurs after extracapsular cataract extraction. Our series helps to fill this gap.

We show that bacterial endophthalmitis continues to be a potentially devastating complication of modern cataract surgery. Five (25\%) culture positive cases failed to regain navigational vision in the eye affected (worse than 6/60). However overall the visual outcome for endophthalmitis following extracapsular cataract surgery compares very favourably with that reported for endophthalmitis occurring in other situations. In addition a good visual outcome may be achieved by 'conventional' treatment alone. Of the 20 culture positive cases $13(65 \%)$ were managed without intravitreal antibiotic or vitrectomy. Only two $(15 \%)$ of these resulted in a final acuity worse than $6 / 60$; both were due to Streptococcus pneumoniae. Eight (62\%) achieved an acuity of $6 / 12$ or better. This relatively favourable outcome may be due to the high proportion of low virulence organisms. However the presence of an intact zonule and posterior capsule may alter the behaviour of an endophthalmitis. Bacteria introduced into the eye during extracapsular cataract surgery may be denied access to the vitreous by the intact capsulo-zonular diaphragm. Such a barrier effect has been demonstrated in primate studies. ${ }^{1920}$ In the human eye the posterior capsule has been shown to be a barrier to the diffusion of fluorescein from the posterior chamber into the vitreous. ${ }^{21}$

Vitreous intervention, whether by vitreous tap, intravitreal antibiotic injection, or vitrectomy, carries a risk of additional ocular morbidity. In our series one rhegmatogenous retinal detachment and three cases of macular pucker followed vitreous tapping and intravitreal antibiotic and each of these cases had a final visual acuity of $6 / 60$ or worse. Gentamicin, either alone or in combination with other antibiotics, was used in every instance of intravitreal antibiotic injection. The retinal toxicity of intravitreal gentamicin has been demonstrated in animal eyes. ${ }^{22}{ }^{23} \mathrm{~A}$ 'safe' dose for injection into 
the human vitreous in the treatment of endophthalmitis has been determined empirically and that currently recommended is $0 \cdot 1 \mathrm{mg} .{ }^{24}$ However much of the published experience of the use of intravitreal gentamicin in cases of endophthalmitis following cataract surgery relates to intracapsular cataract extraction. The presence of an intact posterior capsule may alter the pharmacokinetics of antibiotic injected into the vitreous cavity. The clearance of drug from the vitreous to the anterior chamber may be prolonged. The halflife of a drug injected into the vitreous has been shown to be related to the degree of vitreal inflammation..$^{2+26}$ An intact posterior capsule may delay the establishment of vitreal inflammation in endophthalmitis after extracapsular cataract extraction. The appropriate dose for an intravitreal antibiotic therefore remains uncertain making its use problematic.

The antibiotics traditionally used in ocular infections, when administered topically, subconjunctivally or systemically, attain therapeutic concentrations within the anterior chamber but not within the vitreous..$^{25}$ In an endophthalmitis following uncomplicated extracapsular cataract surgery, where infection is primarily one of the anterior segment, 'conventional' management may be adequate if instigated aggressively and early and where the organism is of low virulence. The use of intravitreal antibiotic injection may be unnecessary and its potential complications thus avoided. Imipenem, a wide spectrum third generation cephalosporin administered intravenously, has been reported to achieve therapeutic levels in both aqueous and vitreous. ${ }^{27}$ Its use may thus further reduce the need for vitreal intervention.

The commonest infecting organism, Staphylococcus epidermidis is associated with a relatively favourable visual outcome. Of our 11 cases nine $(82 \%)$ achieved $6 / 60$ or better with five (45\%) gaining 6/12 or better. O'Day has reviewed Staphylococcus epidermidis endophthalmitis occurring after a variety of surgical procedures and found $78 \%$ achieved a final acuity of $20 / 50$ or better by 'conventional' treatment. ${ }^{13}$ When managed using intravitreal antibiotic and/or vitrectomy only $42 \%$ achieved $20 / 50$ or better. However in all retrospective studies, including ours, there may be a bias towards the worst cases being managed by vitreal intervention.

In our series anterior chamber diagnostic tap was performed in all cases. This yielded a culture positive rate of $59 \%$ (19 of 32). This compares with a $56 \%$ to $77 \%$ culture positive rate for aqueous and/or vitreous tap previously reported in series of cases of endophthalmitis of mixed aetiology. ${ }^{29101226}$ It has been noted that in such mixed cases of endophthalmitis a vitreous tap is more likely to be positive than an anterior chamber tap. ${ }^{3511-14}$ However in the series reported by Driebe et al the only two cases that were anterior chamber culture positive/vitreous tap culture negative were in eyes that had intact posterior capsules and posterior chamber lenses. ${ }^{11}$ In our series five culture positive cases had both anterior chamber and vitreous taps. Four were positive for the anterior chamber tap of which two were also positive for the vitreous tap. One case was culture positive from the vitreous alone (case 11). We therefore consider that in cases of endophthalmitis following uncomplicated extracapsular cataract surgery where the primary focus of infection is in the anterior segment an anterior chamber tap alone is an adequate initial procedure.

In the light of the recently described 'localised endophthalmitis' wherein organisms are sequestered in the capsular bag $^{6}$ we have modified our anterior chamber tap technique. We no longer use a tuberculin type syringe as previously described in the literature. ${ }^{12} 1628 \mathrm{It}$ is our current practice to reopen the cataract section sufficient to insert an 18 gauge coaxial irrigation/aspiration cannula with a $0.3 \mathrm{~mm}$ sideport. With this we take two separate specimens for culture. The first tap is from the anterior chamber, teasing free any fibrinous membranes and including hypopyon where present. The second specimen is taken from within the capsular bag. This may require freeing up of the anterior capsular frill and some intraocular lens manipulation but with care to preserve the integrity of the posterior capsule and zonule. The tap specimens are applied to blood agar, chocolate agar, Sabouraud's media, and thioglycollate broth. The use of an additional enrichment broth, for example brain heart infusion, and specific solid media culture for anaerobes may further increase growth yield. ${ }^{99} 30$ The procedure is concluded with a period of aspiration/irrigation to remove macroscopic debris. Irrigation is with balanced salt solution. The value of this approach is illustrated by case 20 where Staphylococcus epidermidis was cultured from the capsular bag tap while the anterior chamber tap was sterile. Liason with the microbiologist is essential in order than growth of low virulence organisms such as Staphylococcus epidermidis or Propionibacterium is not dismissed as a contaminant.

In no culture positive case did it prove necessary to remove the intraocular lens in order to resolve the infection or facilitate vitrectomy. However in the two culture negative cases with ongoing chronic uveitis a low grade infection within the capsular bag remains a possibility and ultimate removal of the capsular bag and intraocular lens may be necessary to achieve a quiet eye. Two cases (cases 4 and 12) were culture negative on the first anterior chamber tap but culture positive after repeat tap. It is our view therefore that all cases of endophthalmitis after extracapsular cataract surgery should be managed as infections even if culture negative.

It appears to us that endophthalmitis after uncomplicated extracapsular cataract extraction with posterior chamber intraocular lens implantation can be managed by anterior segment diagnostic tap alone and without recourse to vitreal intervention. Delivery of antibiotic by the 'conventional' topical, subconjunctival, and systemic routes is consistent with a favourable visual outcome in many cases and avoids further risks to the eye. It is our current policy only to consider vitreous tap, intravitreal antibiotics, or vitrectomy in cases of endophthalmitis following extracapsular cataract extraction where there 
had been capsular rupture or where there is overt evidence of vitreal infection.

1 Editorial: The endophthalmitis vitrectomy study. Arch Ophthalmol 1991, 109: 487-9.

2 Rowsey JJ, Newsom MS, Sexton DJ, Harms WK. Endophthalmitis: current approaches. Ophthalmology 1982; 89: $1055-66$.

3. Puliafito CA, Baker AS, Haaf J, Foster CS. Infectious endophthalmitis. Ophthalmology 1982; 89: 921-9.

4 Eichenbaum DM, Jaffe NS, Clayman HM, Light DS. Pars plana vitrectomy as a primary treatment for acute bacterial plana vitrectomy as a primary treatment for acute bacte

5 Peyman GA, Raichand M, Bennett TO. Management of endophthalmitis with pars plana vitrectomy. Br $\mathcal{J}$ Ophthal-
enthan mol 1980, 64: 472-5.

6 Carlson AN, Tetz MR, Apple DJ. Infectious complications of modern cataract surgery and intraocular lens implantation. Infect Dis Clin N Am 1989; 3: 339-55.

7 Diamond JG. Intraocular management of endophthalmitis: a systematic approach. Arch Ophthalmol 1981; 99: 96-9.

8 Stern GA, Engel HM, Driebe WT. The treatment of postoperative endophthalmitis: results of differing approaches to treatment. Ophthalmology 1989; 96: 62-7.

9 Bohigian GM, Olk RJ. Factors associated with a poor visual result in endophthalmitis. Am $\mathcal{F}$ Ophthalmol 1986; 101: 33241.

10 Olson JC, Flynn HW, Forster RK, Culbertson WW. Results in the treatment of postoperative endophthalmitis. Ophthalmology 1983; 90: 692-9.

11 Driebe WT, Mandlelbaum S, Forster RK, Schwartz LK, Culbertson WW. Pseudophakic endophthalmitis: diagnosis and management. Ophthalmology 1986; 93: 442-8.

12 Forster RK, Abbott RL, Gelender H. Management of infectious endophthalmitis. Ophthalmology 1980;87: 313-8.

13 O'Day DM, Jones DB, Patrinely J, Elliott JH. Staphylococcus epidermidis endophthalmitis: visual outcome following noninvasive therapy. Ophthalmology 1982; 98: 354-9.

14 Ficker L, Peacock J. Infectious endophthalmitis. Trans Ophthalmol Soc UK 1986; 105: 319-23.

15 Niran VS, Karesh JW, Lakhanpal V, Schocket SS. Pseudophakic endophthalmitis. Ophthalmic Surg 1983; 14: 314-6.

16 Forster RK. Etiology and diagnosis of bacterial postoperative endophthalmitis. Ophthalmology 1978; 85: 320-6.
17 Meisler DM, Palestine AG, Vastine DW, Demartini DR, Murphy BF, Reinhart WJ, et al. Chronic Propionibacterium endophthalmitis after extracapsular cataract extraction and intraocular lens implantation. Am $\mathcal{F}$ Ophthalmol 1986; 102: 733-9.

18 Bode DD, Gelender H, Forster RK. A retrospective review of endophthalmitis due to coagulase-negative staphylococci. Brf Ophthalmol 1985; 69: 915-9.

19 Beyer TL, Sharma D, Vogler G, O'Donnell FE. Protective barrier effect of the posterior lens capsule in exogenous bacterial endophthalmitis: an experimental pseudophakic primate study. Am Intraocul Implant Soc $\mathcal{F}$ 1983; 9: 293-6.

20 Beyer TL, Vogler G, Sharma D, O'Donnell FE. Protective barrier effect of the posterior lens capsule in exogenous bacterial endophthalmitis: an experimental primate study. Invest Ophthalmol Vis Sci 1984; 25: 108-12.

21 Sith RT, Campbell CJ, Koester CJ, Trokel S, Anderson A. The barrier function in extracapsular cataract surgery. Ophthalmology 1990; 97: 90-5.

22 Peyman GA, May DR, Ericson ES, Apple D. Intraocular injection of gentamicin. Arch Ophthalmol 1974; 92 42-7.

23 Brown GC, Eagle RC, Shankin EP, Gruber M, Arbizio VV Retinal toxicity of intravitreal gentamicin. Arch Ophthalmol $1990 ; 108: 1740-4$.

24 Endophthalmitis therapy: changing antibiotic sensitivity patterns and current therapeutic recommendations. (Letter.) Arch Ophthalmol 1991; 109: 175-6.

25 Lesar TS, Fiscella RG. Antimicrobial drug delivery to the eye. Drug Intell Clin Pharm 1985; 19: 642-54

26 Ficker LA, Meredith TA, Gardner S, Wilson LA. Cefazolin levels after intravitreal injection. Invest Ophthalmol Vis $S_{c i}$ 1990; 31: 502-5.

27 Axelrod JL, Newton JC, Klein RM, Bergen RL, Sheikh MZ. Penetration of Imipenem into human aqueous humour and vitreous humour. Am F Ophthalmol 1987; 104: 649-53.

28 Forster RK. Endophthalmitis: diagnostic cultures and visual results. Arch Ophthalmol 1974; 92: 387-92.

29 Sherwood DR, Rich WJ, Jacob S, Hart RJ, Fairchild YL. Bacterial contamination of intraocular and extraocular fluids during extracapsular cataract extraction. Eye 1989; 3: 30812 .

30 Ormerod LD, Paton BG, Haff J, Topping TM, Baker AS. Anaerobic bacterial endophthalmitis. Ophthalmology 1987; 